\title{
ON THE $C^{\star}$-ALGEBRA GENERATED BY THE LEFT REGULAR REPRESENTATION OF A LOCALLY COMPACT GROUP.
}

\author{
by \\ Erik Bédos. \\ Institute of Mathematics \\ University of Oslo \\ P.b. 1053 Blindern \\ 0316 Oslo 3 - Norway
}

\begin{abstract}
Let $\lambda$ denote the left regular representation of a locally compact group $G$ on $L^{2}(G)$ and $C^{\star}(\lambda(G))$ the $C^{\star}$-algebra generated by $\lambda(G)$. We show that the amenability of $G$ and the amenability of $G$ considered as a discrete group may both be characterized in terms of $C^{\star}(\lambda(G))$.
\end{abstract}





\section{Introduction.}

We first fix some notation. Throughout this note we let $G$ denote a locally compact (Hausdorff topological) group equipped with a fixed left Haar measure $\mu$, and $G_{d}$ denote the group $G$ considered as a discrete group. As usual, $L^{1}(G), L^{2}(G)$ and $L^{\infty}(G)$ are defined with respect to $\mu$. The left regular representation of $G$ on $L^{2}(G)$, defined by

$$
\lambda(g) \xi)(h)=\xi\left(g^{-1} h\right), \xi \in L^{2}(G), g, h \in G
$$

is well known to be a (strongly) continuous unitary representation of $G$. We shall denote by $\lambda_{d}$ the left regular representation of $G_{d}$ on $l^{2}\left(G_{d}\right)$. All undefined terminology in this paper is explained in at least one of the following references: [2], [5], [7], [11], [13], [14].

Much attention has been devoted to the study of the following operator algebras associated with $G$ : the full group $C^{\star}$-algebra $C^{\star}(G)$, the reduced group $C^{\star}$-algebra $C_{r}^{\star}(G)$ and the group von Neuman algebra $v N(G)$. We recall that $C^{\star}(G)$ is defined as the enveloping $C^{\star}$-algebra of $L^{1}(G)$ considered as an involutive Banach algebra with an approximate identity. If $\mathcal{B}\left(L^{2}(G)\right)$ denotes the bounded linear operators on $L^{2}(G)$, then $C_{r}^{\star}(G)$ is the $C^{\star}$-subalgebra of $\mathcal{B}\left(L^{2}(G)\right)$ generated by the convolution operators $T_{f}, f \in L^{1}(G)$, where $T_{f}(\xi)=f \star \xi, \xi \in L^{2}(G)$. At last, $v N(G)$ is the von Neumann subalgebra of $\mathcal{B}\left(L^{2}(G)\right)$ generated by $\lambda(G)=\{\lambda(g), g \in G\}$, or equivalently $v N(G)=\lambda(G)^{\prime \prime}=C_{r}^{\star}(G)^{\prime \prime}$, where " denotes the double commutant (in $\mathcal{B}\left(L^{2}(G)\right)$ ). The purpose of this note is to draw the attention 
to $C^{\star}(\lambda(G))$, the $C^{\star}$-subalgebra of $\mathcal{B}\left(L^{2}(G)\right)$ generated by $\lambda(G)$. Of course, when $G$ is discrete, we have $C^{\star}(\lambda(G))=C_{r}^{\star}(G)$, and we will therefore mainly be interested in the non-discrete case. In this case, it is known that $C_{r}^{\star}(G)$ and $C^{\star}(G)$ are non-unital $([10$; Cor. 1 and 2$])$, while $C^{\star}(\lambda(G))$ is always unital.

The only paper we are aware of which explicitely deals with $C^{\star}(\lambda(G))$ in the non-discrete case is [8], where Kodaira and Kakutani essentially show that when $G$ is abelian, then $C^{\star}(\lambda(G))$ is $\star$-isomorphic to $\mathcal{C}\left(\widehat{G_{d}}\right)$, the continuous complex functions on the dual group of $G_{d}$. This result is nicely exposed by Arveson in [1], where he generalizes it to other $C^{\star}$-algebras generated by abelian unitary groups. Further, when $G$ is abelian, it is well known that $C^{\star}(G) \simeq C_{r}^{\star}(G) \simeq \mathcal{C}_{o}(\widehat{G})$, the continuous complex functions on the dual group of $G$ which vanish at infinity. Thus, $C^{\star}(\lambda(G))$ on one hand and $C^{\star}(G) \simeq C_{r}^{\star}(G)$ on the other hand contain rather different information in the abelian case. However, still in this case, we also have $C_{r}^{\star}\left(G_{d}\right) \simeq \mathcal{C}\left(\widehat{G_{d}}\right)$, hence $C^{\star}(\lambda(G)) \simeq C_{r}^{\star}\left(G_{d}\right)$, which shows that the topology of $G$ is not reflected in $C^{\star}(\lambda(G))$. One may therefore wonder whether all the topological flavour of $G$ does disappear in $C^{\star}(\lambda(G))$ in the non-abelian case too.

We shall show that this suggestion is not generally true. Our approach relies heavily on the now well-developped theory of amenability ([13], [14]). We recall that $G$ is called amenable whenever there exists a left invariant mean on $L^{\infty}(G)$, i.e. a state on $L^{\infty}(G)$ which is invariant under left translations. A deep $C^{\star}$-algebraic characterization of the amenability of $G$ is that $C^{\star}(G)$ 
and $C_{r}^{\star}(G)$ are canonically $\star$-isomorphic. ([12; Theorem 4.21$]$ or [13; Theorem 8.9]). Another characterization via $C^{\star}(\lambda(G))$ is possible: our first result (Theorem 1) is that $G$ is amenable if and only if there exists a non zero multiplicative linear functional on $C^{\star}(\lambda(G))$. We notice that the "only if" part is known in the discrete case ([3; Theorem 2], [12; Proof of prop. 1.6]). This result provides a natural $C^{\star}$-explanation to the fact that an abelian group $G$ is amenable: $C^{\star}(\lambda(G))$ is then an abelian $C^{\star}$-algebra and therefore possess a non-zero multiplicative linear functional by Gelfand's theory. Of course, this is not the most efficient way to prove this fact which is an easy consequence of the Markov-Kakutani fixed point theorem (cf. [13; Proposition 0.15]).

By combining a remark of Arveson in [1] and some arguments of FigàTalamanca in [6], one obtains that if $G_{d}$ is amenable, then $C^{\star}(\lambda(G)) \simeq$ $C_{r}^{\star}\left(G_{d}\right)$. With the help of Theorem 1, we can conclude that $G_{d}$ is amenable if and only if $G$ is amenable and $C^{\star}(\lambda(G)) \simeq C_{r}^{\star}\left(G_{d}\right)$. (Theorem 2). Hence, if $G$ is an amenable group such that $G_{d}$ is not amenable (f.ex. $G=S O(3)$ ), then $C^{\star}(\lambda(G))$ is not $\star$-isomorphic to $C_{r}^{\star}\left(G_{d}\right)$.

At last, we characterize the nuclearity of $C^{\star}(\lambda(G))$. We recall that a $C^{\star_{-}}$ algebra is called nuclear if there is a unique way of forming its tensor product with any other $C^{\star}$-algebra. For some equivalent definitions, the reader may consult [9], [13] or [15] where further references are given. As a sample of the work of many hands, we quote the following from [13; 1.31 and 2.35]: $G$ is amenable if and only if $G$ is inner amenable and $C_{r}^{\star}(G)$ is nuclear, if and only if $G$ is inner amenable and $v N(G)$ is injective. 
Inner amenability of $G$ means here that there exists a state on $L^{\infty}(G)$ invariant under the action on $L^{\infty}(G)$ by inner automorphisms of $G$, while $v N(G)$ is injective whenever there exists a norm one projection from $\mathcal{B}\left(L^{2}(G)\right)$ onto $v N(G)$. We also recall that there exist non-amenable groups $G$ such that $C_{r}^{\star}(G)$ is nuclear and $v N(G)$ is injective. Now, since any discrete group is inner amenable in the above sense, we have $G_{d}$ is amenable if and only if $C_{r}^{\star}\left(G_{d}\right)$ is nuclear, a result proved by Lance in [9; Theorem 4.2]. We shall use this to conclude that $G_{d}$ is amenable if and only if $C^{\star}(\lambda(G))$ is nuclear (Theorem 2). Especially, we get that if $G$ is amenable but $G_{d}$ is not, then $C^{\star}(\lambda(G))$ is non-nuclear while $C_{r}^{\star}(G)$ is nuclear and $v N(G)$ is injective.

\section{The results.}

We begin with a lemma which is surely known to specialists, but for the convenience of the reader we sketch the proof.

Lemma A: Let $\mathcal{A}$ denote a unital $C^{\star}$-algebra, $\mathcal{U}(\mathcal{A})$ its unitary group and $\varphi$ a state on $\mathcal{A}$. Let $x \in \mathcal{A}$ and $u \in \mathcal{U}(\mathcal{A})$. Then

a) $\varphi(x a)=\varphi(x) \varphi(a)$ for all $a$ in $\mathcal{A}$ if and only if $\varphi\left(x x^{\star}\right)=|\varphi(x)|^{2}$.

b) $\varphi(a x)=\varphi(a) \varphi(x)$ for all $a$ in $\mathcal{A}$ if and only if $\varphi\left(x^{\star} x\right)=|\varphi(x)|^{2}$.

c) $\varphi(u a)=\varphi(a u)=\varphi(u) \varphi(a)$ for all $a$ in $\mathcal{A}$ if and only if $|\varphi(u)|=1$.

d) If $\mathcal{V}$ is a subgroup of $\mathcal{U}(\mathcal{A})$ which generates $\mathcal{A}$ as a $C^{\star}$-algebra, then $\varphi$ is multiplicative if and only if $|\varphi(v)|=1$ for all $v$ in $\mathcal{V}$. 


\section{Proof:}

a) Suppose $\varphi\left(x x^{\star}\right)=|\varphi(x)|^{2}$ and let $a \in \mathcal{A}$. Then, by the CauchySchwartz inequality, we get

$$
\begin{aligned}
|\varphi(x a)-\varphi(x) \varphi(a)|^{2} & =|\varphi((x-\varphi(x)) a)|^{2} \\
& \leq \varphi\left(a^{\star} a\right) \varphi\left((x-\varphi(x))(x-\varphi(x))^{\star}\right) \\
& =\varphi\left(a^{\star} a\right)\left(\varphi\left(x x^{\star}\right)-|\varphi(x)|^{2}\right) \\
& =0 .
\end{aligned}
$$

Hence $\varphi(x a)=\varphi(x) \varphi(a)$ as desired.

The only if part is trivial.

b) may be deduced from a) or proved similarly.

c) follows from a) and b).

d) follows from c) and an easy density argument.

Theorem 1: $G$ is amenable if and only if there exists a non-zero multplicative linear functional on $C^{\star}(\lambda(G))$.

Proof: Suppose $G$ is amenable. Then there exists a net $\left\{\xi_{i}\right\}$ in $\left\{\xi \in L^{2}(G) \mid\|\xi\|_{2}=1\right\}$ such that

$$
\left\|\lambda(g) \xi_{i}-\xi_{i}\right\|_{2} \rightarrow 0 \text { for all } g \text { in } G
$$


(cf. [13; Theorem 4.4] or [14; Corollary 6.15]). For each $i$, define $\varphi_{i}$ on $C^{\star}(\lambda(G))$ by

$$
\varphi_{i}(x)=<x \xi_{i}, \xi_{i}>, \quad x \in C^{\star}(\lambda(G)) .
$$

Then $\left\{\varphi_{i}\right\}$ is a net in the state space of $C^{\star}(\lambda(G))$ which (by Banach-Alaoglu's theorem) is weak ${ }^{\star}$-compact. Hence we may pick a weak ${ }^{\star}-$ limit point of this net, say $\varphi$, which is a state on $C^{\star}(\lambda(G))$. Now, since

$$
\begin{aligned}
\left|\varphi_{i}(\lambda(g))-1\right|^{2} & =\left|<\left(\lambda(g) \xi_{i}-\xi_{i}\right), \xi_{i}>\right|^{2} \\
& \leq\left\|\lambda(g) \xi_{i}-\xi_{i}\right\|_{2} \rightarrow 0 \text { for all } g \text { in } G
\end{aligned}
$$

we clearly have $\varphi(\lambda(g))=1$ for all $g$ in $G$. As $\lambda(G)$ generates $C^{\star}(\lambda(G))$ by definition, it follows from lemma $\mathrm{A} \mathrm{d}$ ) that $\varphi$ is a non-zero multiplicative linear functional on $C^{\star}(\lambda(G))$.

Conversly, suppose $\varphi$ is such a functional on $C^{\star}(\lambda(G))$. Then, as $\varphi$ preserves adjoints ([11; Prop. 2.1.9 ]), $\varphi$ is a state on $C^{\star}(\lambda(G)$ ) such that $|\varphi(\lambda(g))|=1$ for all $g$ in $G$. By the Hahn-Banach theorem for states ([2; Prop. 2.3.24]), we may extend $\varphi$ to a state $\tilde{\varphi}$ on $\mathcal{B}\left(L^{2}(G)\right)$ which satisfies

$$
|\tilde{\varphi}(\lambda(g))|=1 \text { for all } g \text { in } G .
$$

As a consequence of lemma $\mathrm{A} \mathrm{c}$ ), we then have

$$
\begin{aligned}
\tilde{\varphi}\left(\lambda(g) x \lambda\left(g^{-1}\right)\right) & =\tilde{\varphi}(\lambda(g)) \tilde{\varphi}\left(x \lambda\left(g^{-1}\right)\right) \\
& =\tilde{\varphi}(\lambda(g)) \tilde{\varphi}(x) \tilde{\varphi}\left(\lambda\left(g^{-1}\right)\right) \\
& =|\tilde{\varphi}(\lambda(g))|^{2} \tilde{\varphi}(x) \\
& =\tilde{\varphi}(x)
\end{aligned}
$$


for all $g$ in $G$ and $x$ in $\mathcal{B}\left(L^{2}(G)\right)$.

The amenability of $G$ follows readily from this in a quite standard way. If $M_{f}$ denotes the multiplication operator on $L^{2}(G)$ by $f \in L^{\infty}(G)$, then one obtains a left invariant mean $m$ on $L^{\infty}(G)$ by defining $m(f)=\tilde{\varphi}\left(M_{f}\right), f \in$ $L^{\infty}(G)$, and using that $M_{f_{g}}=\lambda(g) M_{f} \lambda\left(g^{-1}\right)$ for all $f$ in $L^{\infty}(G)$ and $g$ in $G$, where $f_{g}(h)=f\left(g^{-1} h\right), h \in G$.

When $U$ is a continuous unitary representation of $G$ on a Hilbert space $\mathcal{H}$, we denote by $\pi_{U}$ the canonically associated $\star$ representation of $C^{\star}(G)$ in $\mathcal{B}(\mathcal{H})$. We recall that if $V$ is such another representation of $G$, then $U$ is said to be weakly contained in $V$ (resp. equivalent to $V$ ) whenever $\operatorname{ker} \pi_{V} \subseteq \operatorname{ker} \pi_{U}$ (resp. $\left.\operatorname{ker} \pi_{V}=\operatorname{ker} \pi_{U}\right)$. We shall also need the fact that $\pi_{U}\left(C^{\star}(G)\right)$ is the closure (in the uniform topology) of $\pi_{U}\left(L^{1}(G)\right)$ in $\mathcal{B}(\mathcal{H})$. We refer to [5] for more information on this matter.

By regarding $G$ as a discrete group, we may consider $\lambda$ as a representation of $G_{d}$ in $L^{2}(G)$. To avoid confusion, we shall denote this representation by $\lambda^{\circ}$. For each $g \in G$, we let $\delta_{g}$ denote the characteristic function of $\{g\}$ in $G$.

Lemma B: $\quad C^{\star}(\lambda(G))=\pi_{\lambda^{\circ}}\left(C^{\star}\left(G_{d}\right)\right)$.

Proof: Let $\xi, \eta \in L^{2}(G)$. Then for all $g$ in $G$ we have

$$
\begin{aligned}
<\pi_{\lambda^{\circ}}\left(\delta_{g}\right) \xi, \eta> & =\sum_{h \in G} \delta_{g}(h)<\lambda^{\circ}(h) \xi, \eta>=<\lambda^{\circ}(g) \xi, \eta> \\
& =<\lambda(g) \xi, \eta>
\end{aligned}
$$


Hence $\pi_{\lambda^{\circ}}\left(\delta_{g}\right)=\lambda(g), g \in G$. This clearly implies that $C^{\star}(\lambda(G)) \subseteq$ $\pi_{\lambda^{\circ}}\left(C^{\star}\left(G_{d}\right)\right)$. To prove the converse inclusion, let $f \in l^{1}\left(G_{d}\right)$. Then choose a sequence of complex functions $f_{n}$ with finite support such that $f_{n} \rightarrow f$ in $l^{1}$-norm. From the above, we have $\pi_{\lambda^{\circ}}\left(f_{n}\right) \in C^{\star}(\lambda(G))$ for all $n$. Since

$$
\begin{aligned}
\left\|\pi_{\lambda^{\circ}}\left(f_{n}\right)-\pi_{\lambda^{\circ}}(f)\right\| & =\left\|\pi_{\lambda^{\circ}}\left(f_{n}-f\right)\right\| \\
& \leq\left\|f_{n}-f\right\|_{1} \rightarrow 0
\end{aligned}
$$

we get $\pi_{\lambda^{\circ}}(f) \in C^{\star}(\lambda(G))$.

Thus $\pi_{\lambda^{\circ}}\left(l^{1}\left(G_{d}\right)\right) \subseteq C^{\star}(\lambda(G))$, so

$$
\left.\pi_{\lambda^{\circ}}\left(C^{\star}\left(G_{d}\right)\right)=\overline{\pi_{\lambda^{\circ}}\left(l^{1}\left(G_{d}\right)\right)}\right)^{\|\cdot\|} \subseteq C^{\star}(\lambda(G))
$$

The next lemma is a corollary of [1] and [6], but for the sake of completeness, we sketch the proof.

Lemma C: $\lambda_{d}$ is weakly contained in $\lambda^{\circ}$. Further, if $G_{d}$ is amenable, then $\lambda_{d}$ is weakly equivalent to $\lambda^{\circ}$ and $C^{\star}(\lambda(G))$ is $\star$-isomorphic to $C_{r}^{\star}\left(G_{d}\right)$.

Proof: For each finite subset $F$ of $G$, there exists a $\xi_{F}$ in $L^{2}(G)$ such that $\left\|\xi_{F}\right\|_{2}=1$ and $\left\langle\lambda(g) \xi_{F}, \xi_{F}>=0\right.$ for all $g$ in $F, g \neq e$ (the identity of $G)$. This follows from the easily verified fact that there exists a Borel subset $W=W(F)$ of $G$ such that $0<\mu(W)<\infty$ and $\mu(g W \cap W)=0$ for all $g$ in $F, g \neq e$, and then by setting $\mu(W)^{1 / 2} \cdot \xi_{F}=\chi_{W}$ (the characteristic function of $W$ ). 
Define so $\varphi_{F}(g)=<\lambda(g) \xi_{F}, \xi_{F}>=<\lambda^{\circ}(g) \xi_{F}, \xi_{F}>$ for each $g$ in $G$. Then $\varphi_{F}$ a positive definite function on $G_{d}$ associated to $\lambda^{\circ}$. Further, if we regard $\{F \subseteq G, F$ finite $\}$ as a directed set ordered by inclusion, then we clearly have

$$
\varphi_{F}(g) \rightarrow \delta_{e}(g) \text { for all } g \text { in } G
$$

Since $\delta_{e}(g)=\left\langle\lambda_{d}(g) \delta_{e}, \delta_{e}>\right.$ for all $g$ in $G, \delta_{e}$ is a positive definite function on $G_{d}$ associated to $\lambda_{d}$. As $\delta_{e}$ is a cyclic vector for $\lambda_{d}$, we then get from [5; Prop. 18.1.4] that $\lambda_{d}$ is weakly contained in $\lambda^{\circ}$ as desired.

Now, suppose $G_{d}$ is amenable. Then $\rho$ is weakly contained in $\lambda_{d}$ for all unitary representations $\rho$ of $G_{d}$ (use [5; Prop. 18.3.5] together with [5; Prop. 18.3.6] or [14; Theorem 8.9]). Especially, $\lambda^{\circ}$ is then weakly contained in $\lambda_{d}$. Hence $\lambda_{d}$ is weakly equivalent to $\lambda^{\circ}$.

Since $C_{r}^{\star}\left(G_{d}\right)=\pi_{\lambda_{d}}\left(C^{\star}\left(G_{d}\right)\right)$ and

$$
\left.C^{\star}(\lambda(G))=\pi_{\lambda^{\circ}}\left(C^{\star}\left(G_{d}\right)\right) \text { (by lemma } B\right)
$$

this implies that $C^{\star}(\lambda(G)) \simeq C_{r}^{\star}\left(G_{d}\right)$.

Theorem 2: The following statements are equivalent:

(i) $G_{d}$ is amenable.

(ii) $G$ is amenable and $C^{\star}(\lambda(G)) \simeq C_{r}^{\star}\left(G_{d}\right)$.

(iii) $C^{\star}(\lambda(G))$ is nuclear.

(iv) $C_{r}^{\star}\left(G_{d}\right)$ is nuclear. 
Proof: $\quad \underline{\text { (i) } \Leftrightarrow \text { (iv) }}$ is proved by Lance in [9; Theorem 4.2$]$.

(i) $\Rightarrow$ (ii) Suppose $G_{d}$ is amenable. Then $G$ is amenable ([13; Problem 1.12] or [14; Prop. 4.21]) and $C^{\star}(\lambda(G)) \simeq C_{r}^{\star}\left(G_{d}\right)$ by lemma B.

(ii) $\Rightarrow$ (i) Suppose $G$ is amenable and $C^{\star}(\lambda(G)) \simeq C_{r}^{\star}\left(G_{d}\right)$. From Theorem 1, we then know that $C^{\star}(\lambda(G))$ possess a nonzero multiplicative linear functional, and therefore that $C_{r}^{\star}\left(G_{d}\right)$ possess one too. Since $C_{r}^{\star}\left(G_{d}\right)=$ $C^{\star}\left(\lambda_{d}\left(G_{d}\right)\right)$, Theorem 1 now implies that $G_{d}$ is amenable.

(iii) $\Rightarrow$ (iv) Suppose $C^{\star}(\lambda(G))$ is nuclear. Since $\lambda_{d}$ is weakly contained in $\lambda^{\circ}$ by lemma B, this implies that $\pi_{\lambda_{d}}\left(C^{\star}\left(G_{d}\right)\right)=C_{r}^{\star}\left(G_{d}\right)$ is a quotient $C^{\star}$-algebra of $\pi_{\lambda^{\circ}}\left(C^{\star}\left(G_{d}\right)\right)=C^{\star}(\lambda(G))$. As it is known that a quotient $C^{\star}$-algebra of a nuclear $C^{\star}$-algebra is itself nuclear $([4 ;$ Corollary 4$])$, we obtain that $C_{r}^{\star}\left(G_{d}\right)$ is nuclear.

(iv) $\Rightarrow$ (iii) Suppose $C_{r}^{\star}\left(G_{d}\right)$ is nuclear. Since we now know that

(iv) $\Rightarrow$ (ii), we have $C^{\star}(\lambda(G)) \simeq C_{r}^{\star}\left(G_{d}\right)$, so $C^{\star}(\lambda(G))$ is nuclear too.

We conclude this note with some remarks on

$$
X(G)=\left\{\varphi: C^{\star}(\lambda(G)) \rightarrow \mathbf{C} \mid \varphi \text { is nonzero, linear and multiplicative }\right\}
$$

which is a weak ${ }^{\star}$-closed subset of the state space of $C^{\star}(\lambda(G))$. Theorem 1 says that $X(G) \neq \phi$ if and only if $G$ is amenable. When $G$ is abelian, the result of Kodaira and Kakutani mentionned in the introduction may be interpreted as the fact that $X(G)$ is homeomorphic to $\widehat{G_{d}}$. In the nonabelian case, $X(G)$ is of course a rather primitive $C^{\star}$-algebraic invariant for 
$C^{\star}(\lambda(G))$, but it has the advantage of being easily computed in some cases, as the following illustrates.

Let $H$ denote a discrete group and $\mathrm{CH}$ its commutator subgroup. Then $H / C H$ is abelian and it is not difficult to show, as it has been observed by Watatani in [16], that if $H$ is amenable, then $X(H)$ is homeomorphic to $H \widehat{T C} H$. Hence, if $G_{d}$ is amenable, we get via Theorem 2 that $X(G)$ is homeomorphic to $G_{d} \widehat{C C} G_{d}$. If $G$ is amenable but $G_{d}$ is not, one can show that $X(G)$ contains a copy of $G \widehat{\widetilde{C C} G}$ and may itself be embedded in $G_{d} \widehat{/ C} G_{d}$, but we don't know whether anything more general can be said here. If f.ex. $G=S O(3)$, then $C G_{d}=G_{d}$, so $X(G)=\{\hat{1}\}$ (where $\hat{1}$ denotes the state on $C^{\star}(\lambda(G))$ determined by $\hat{1}(\lambda(g))=1$ for all $g$ in $G$, cf. the proof of Theorem 1). 


\section{REFERENCES:}

1. W.B.Arveson: A theorem on the action of abelian unitary groups. Pacific J. of Math. 16 (1966), 205-212.

2. O.Bratteli and D.W.Robinson: Operator algebras and quantum statistical mechanics vol. I, Springer-Verlag New York Inc. (1979).

3. H.Choda and M.Choda: Fullness, simplicity and inner amenability. Math. Jap. 24 (1979), 235-246.

4. M.D.Choi and E.G.Effros: Nuclear $C^{\star}$-algebras and injectivity; the general case. Indiana Univ.Math.J. 26 (1977), 443-446.

5. J.Dixmier: Les $C^{\star}$-algèbres et leurs représentations ( $2 \frac{\text { ème }}{}$ ed). GauthierVillars, Paris (1969).

6. A.Figà-Talamanca: On the action of unitary groups on a Hilbert space. Symposia Math. XXII (1977), 314-319.

7. E.Hewitt and K.A.Ross: Abstract Harmonic analysis vol. I, SpringerVerlag, Berlin. Göttingen. Heidelberg. (1963).

8. K.Kodaira and S.Kakutani: Normed ring of a locally compact abelian group. Proc.Imp.Acad. Tokyo 19 (1943), 360-365.

9. E.C.Lance: On nuclear $C^{\star}$-algebras. Journ. of Funct. Anal. 12 (1973), $157-176$. 
10. P.Milnes: Identities of group algebras. Proc.Amer.Math.Soc. 29 (1971), $421-422$.

11. G.Murphy: Operator theory and $C^{\star}$-algebras. Academic Press (1990).

12. W.L.Paschke and N.Salinas: $C^{\star}$-algebras associated with the free product of groups. Pacific J.Math. 82 (1979), 211-221.

13. A.L Paterson: Amenability. Math.Survey and Monographs 29. Amer.Math.Soc., Providence, Rhode Island (1988).

14. J.P.Pier: Amenable locally compat groups. John Wiley and Sons, New York (1984).

15. A.M.Torpe: Notes on nuclear $C^{\star}$-algebras and injective von Neumann algebras. Preprint (1981), Mat.Inst., Odense Universitet.

16. Y.Watatani: The character group of amenable group $C^{\star}$-algebras. Math.Jap. 24 (1979), 141-144. 
